Case Report

\title{
Acute Gastrointestinal Bleed from a Gastrointestinal Stromal Tumour Impersonating an Arteriovenous Malformation of the Jejunum
}

\author{
Oshan Basnayake $\mathbb{D}^{1},{ }^{1}$ Umesh Jayarajah ${ }^{(D)}{ }^{1}$ Nilesh Fernandopulle, ${ }^{2}$ \\ and Sivasuriya Sivaganesh $\mathbb{1}^{2}$ \\ ${ }^{1}$ Professorial Surgical Unit, National Hospital of Sri Lanka, Colombo, Sri Lanka \\ ${ }^{2}$ Department of Surgery, Faculty of Medicine, University of Colombo, Sri Lanka \\ Correspondence should be addressed to Sivasuriya Sivaganesh; sivaganesh@srg.cmb.ac.lk
}

Received 4 October 2020; Revised 4 January 2021; Accepted 9 January 2021; Published 18 January 2021

Academic Editor: Eric Bergeron

Copyright (C) 2021 Oshan Basnayake et al. This is an open access article distributed under the Creative Commons Attribution License, which permits unrestricted use, distribution, and reproduction in any medium, provided the original work is properly cited.

\begin{abstract}
Gastrointestinal stromal tumours (GIST) are neoplasms which originate from the mesenchymal tissue of the gastrointestinal tract. We report on a GIST presenting with acute gastrointestinal bleeding that mimicked an arteriovenous malformation (AVM) on imaging and at surgery. A 61-year-old female presented with a short history of melaena and severe anaemia. After resuscitation, upper gastrointestinal endoscopy showed a profusely bleeding varix in the proximal jejunum which was treated with glue injection. Contrast-enhanced CT showed a heterogeneous lesion in the proximal jejunum with strong arterial phase enhancement, supplied by a branch of the superior mesenteric artery and drained by a prominent tributary of the superior mesenteric vein, suggestive of an AVM. The mass was resected, and histology revealed a GIST with no evidence of an AVM or angiodysplasia. A GIST may be considered, though imaging suggests a diagnosis of an AVM in patients presenting with acute gastrointestinal bleeds. In such circumstances, surgical resection and pathological assessment will be confirmatory.
\end{abstract}

\section{Introduction}

Gastrointestinal stromal tumours are a subset of neoplasms which originate from the mesenchymal tissue of the gastrointestinal tract. They differ from other mesenchymal neoplasms such as leiomyomas, leiomyosarcomas, and spindle cell tumours [1]. Reported incidence of GISTs ranges from 0.55 to 0.78 per 100,000 with a male predominance [2]. However, the presentation of a GIST mimicking an arteriovenous malformation (AVM) is rare and only few cases have been reported [3-6]. We describe a 61-year-old female with an unusual presentation of a GIST as an AVM.

\section{Case Presentation}

A 61-year-old female with hypertension and dyslipidaemia presented with the passage of altered blood of 4-day duration. This was the first episode, and her bowel habits had been normal to date. On admission, she was pale with a pulse rate of 118 per minute and a blood pressure of $86 / 66 \mathrm{mmHg}$. Her haemoglobin was $6.2 \mathrm{~g} / \mathrm{dl}$, and her coagulation profile, liver functions, and renal functions were normal.

She was resuscitated initially with crystalloids and then packed red cell transfusions. The next day, she underwent upper gastrointestinal endoscopy (UGIE) which revealed a protruding mass with active bleeding in the distal duodenum. Upper gastrointestinal assessment was repeated in the same setting using a paediatric colonoscope, and a profusely bleeding varix was observed in the proximal jejunum (Figure 1). Haemostasis was achieved by glue injection. A colonoscopy was also performed in the same setting which was normal. Abdominal ultrasonography was unremarkable. On day 6 after admission, a contrast CT of the abdomen was performed. It showed a welldefined, exophytic, lobular lesion with mixed density in proximal jejunum measuring $3 \times 4 \times 4 \mathrm{~cm}$ (Figure 2). The 


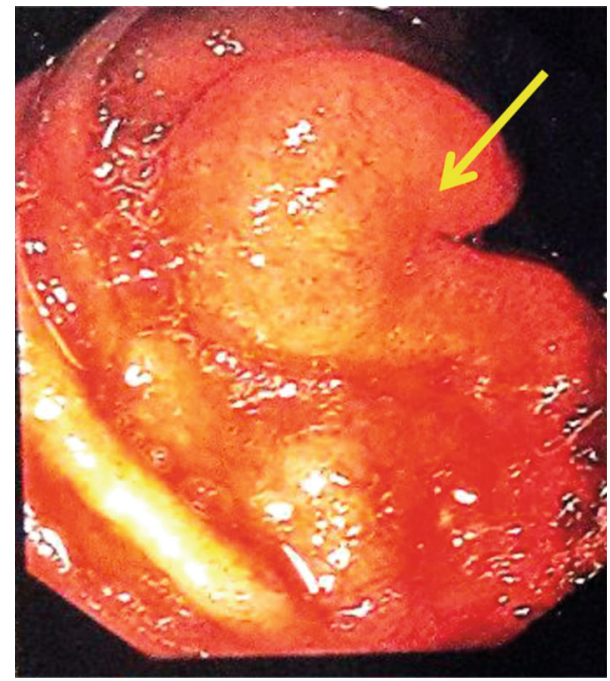

Figure 1: Yellow arrow shows the endoscopic appearance of bleeding varix at proximal jejunum.

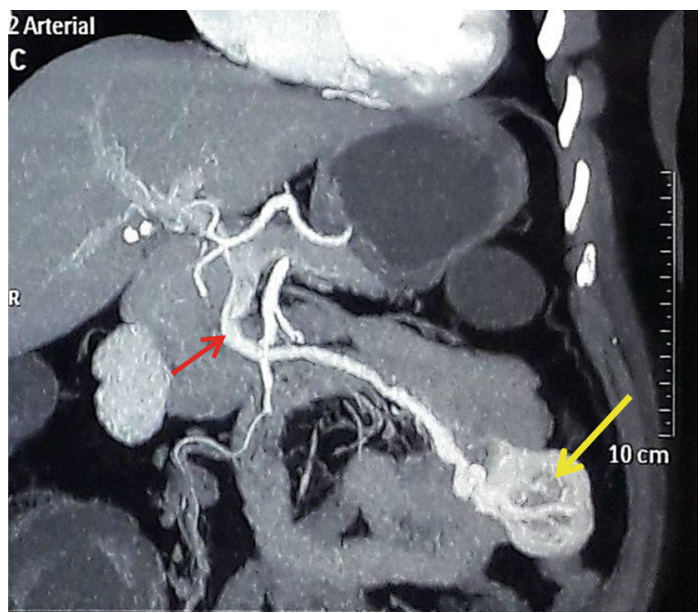

Figure 2: Coronal view of contrast-enhanced CT scan image arterial phase: yellow arrow shows the jejunal lesion; red arrow shows the contrast enhancement of superior mesenteric vein.

lesion displayed strong arterial phase enhancement, mainly at the periphery, and was supplied by a branch from the superior mesenteric artery and drained by a prominent tributary of the superior mesenteric vein just proximal to the portal venous confluence. Arterial phase enhancement of the draining vein, superior mesenteric (SMV), and portal vein (PV) was indicative of an AVM. The facility for magnetic resonance imaging (MRI) is very limited in our resource-limited setting, and therefore, we did not proceed with an MRI for further delineation of the lesion as the CT images were sufficiently conclusive.

Surgery was carried out in the index hospitalization while the patient was stable, in order to avoid a risk of rebleeding. Laparotomy revealed a mass with two prominent feeder vessels at the antemesenteric border of the proximal jejunum, $10 \mathrm{~cm}$ distal to the duodenojejunal flexure (Figures 3-5). A segmental jejunal resection including the mass was per-

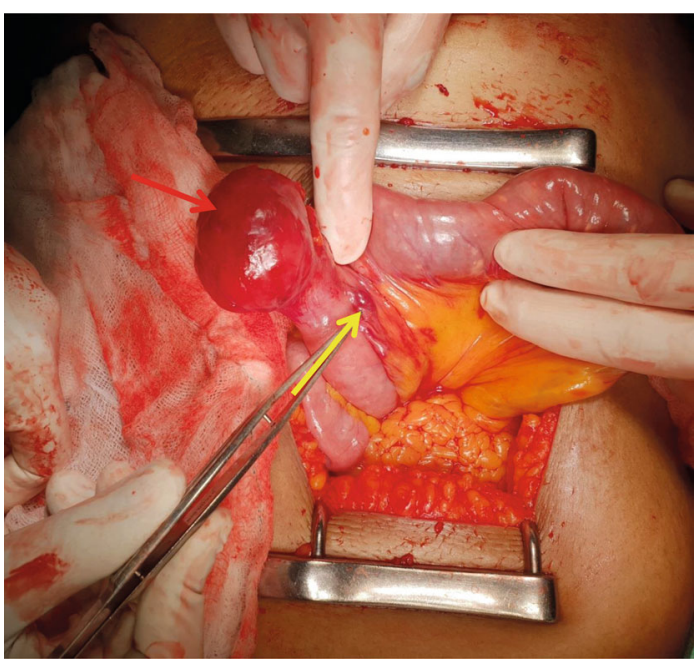

FIGURE 3: Intraoperative image: red arrow shows the jejunal lesion; yellow arrow shows the main feeding vessel through mesentery.

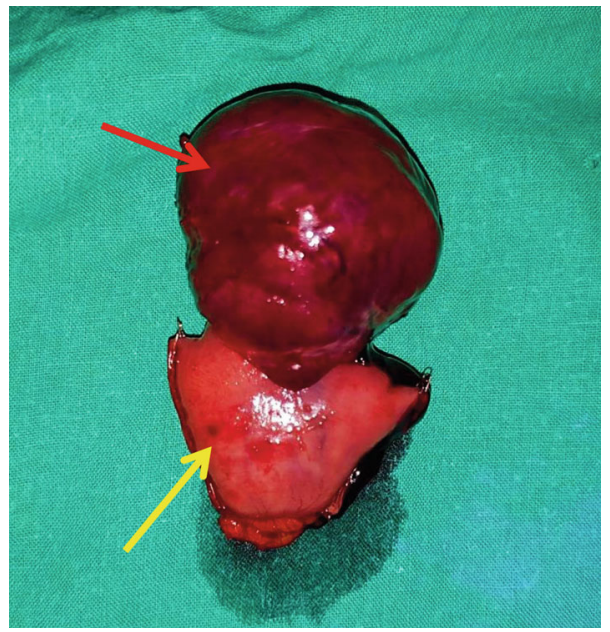

FIgURE 4: Resected specimen: red arrow shows the jejunal lesion; yellow arrow shows part of the jejunum.

formed, and continuity was restored with a side to side anastomosis. Histology revealed a gastrointestinal stromal tumour (GIST) composed of short fascicles of spindle cells. There was no histological evidence of an AVM or angiodysplasia. Her postoperative recovery was uneventful. She was referred to the oncologist and received adjuvant oral imatinib therapy.

\section{Discussion}

GISTs occur along the length of the gastrointestinal tract, the stomach, and the small intestine being the commonest locations [7]. Tumour characteristics and behaviour vary between individual neoplasms, and current consensus is based on risk of recurrence and metastasis. Clinical presentation spans from incidental detection to massive gastrointestinal bleeding. The common submucosal location 


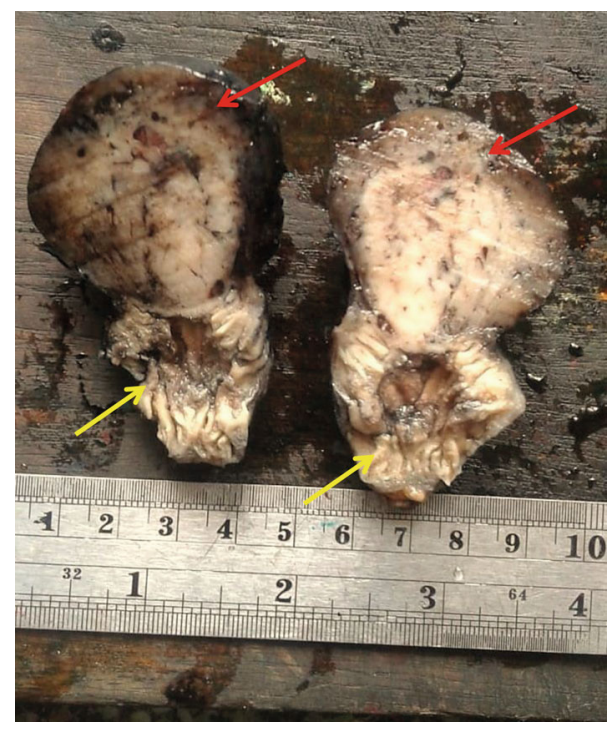

FIGURE 5: Macroscopic appearance of the tumour cross-section: red arrows show the jejunal lesion arising from the wall; yellow arrows show the jejunal lumen.

TABLE 1: Summary of previous case reports that described a similar presentation.

\begin{tabular}{|c|c|c|c|c|c|}
\hline Author & $\begin{array}{c}\text { Year of } \\
\text { publication }\end{array}$ & $\begin{array}{c}\text { Age } \\
\text { (years) }\end{array}$ & Presentation & $\begin{array}{l}\text { Site of } \\
\text { GIST }\end{array}$ & Histology \\
\hline 1. Tomita et al. [3] & 2004 & 47 & Melaena & Jejunum & $\begin{array}{l}\text { Submucosal angiodysplasia } \\
\text { with spindle cell type GIST }\end{array}$ \\
\hline Shim et al. [4] & 2008 & 44 & Melaena & Jejunum & $\begin{array}{c}\text { Spindle cell type GIST and AVM located } \\
\text { in the overlying submucosa and } \\
\text { muscularis propria }\end{array}$ \\
\hline 3. Shiozawa et al. [5] & 2011 & 62 & $\begin{array}{l}\text { Intermittent left upper } \\
\text { abdominal pain }\end{array}$ & Jejunum & $\begin{array}{l}\text { Spindle cell type GIST without } \\
\text { histological features of AVM }\end{array}$ \\
\hline 4. Javeed et al. [6] & 2015 & 23 & Melaena and anaemia & Jejunum & $\begin{array}{c}\text { Spindle cell type GIST and was intermingled } \\
\text { with the AVM }\end{array}$ \\
\hline
\end{tabular}

causes overlying mucosal ulceration and bleeding which ranges from occult to life-threatening overt bleeding [8]. The diagnosis relies on contrast CT, endoscopy, and endosonography-guided biopsy [9]. The CT scan finding of a well-defined mass with homogenous contrast enhancement is characteristic of GIST with a sensitivity and specificity of $94.9 \%$ and $100 \%$, respectively $[10,11]$. However, GISTs mimicking an arteriovenous malformation are uncommon. Imaging alone would not be useful in differentiation, and diagnosing a GIST and magnetic resonance imaging (MRI) scans do not offer any additional benefit [9]. Contrast enhancement of the draining vein of the mass along with the SMV and PV (Figure 2) was suggestive of an AVM. Only 4 such cases have been reported worldwide with similar presentation as summarized in Table 1.

The misinterpretation of AV malformation in our patient was based on the hypervascularity of the tumour with the superior mesenteric vein enhancement in the arterial phase of the CT which was comparable to the contrast enhancement of the superior mesenteric artery and the aorta. Similarly, all reported cases were in the jejunum and CT scans showed contrast enhancement of veins during the arterial phase suggestive of an AVM or angiodysplasia. Three of the reported cases had an associated AVM in histology $[3,4,6]$. Similar to the case reported by Shiozawa et al. [5], there was no histological evidence of an AVM or angiodysplasia in our patient. Vats et al. also reported another patient with a similar presentation found to have a Dieulafoy lesion overlying a gastric GIST without histological features of an AVM or angiodysplasia [12].

Treatment of patients with bleeding GISTs or AVMs depends on the initial presentation and the severity of bleeding. Endoscopic methods are useful in accessible regions, though the rebleeding risk after endoscopic treatment alone is significant, especially in angiodysplastic lesions [13]. In our patient, endoscopic injection of glue allowed stabilisation of the condition before planning surgery similar to angiography and embolization. Surgery was delayed until the CT scan was performed after day 6 of admission as there were logistic issues in performing the CT scan in our resource-limited setting. Differentiation between a vascular malformation and a GIST is useful because segmental resection with clear margins is warranted in all potentially resectable GISTs [14]. However, 
although important to be differentiated, surgery is also the treatment in the localized AVF involving the bowel.

\section{Conclusion}

We report an unusual presentation of a GIST mimicking an AVM. The possibility of a coexistent or masquerading GIST should be considered when the diagnosis of an AVM is made in patients presenting with gastrointestinal bleeds. In such circumstances, surgical resection and histological confirmation are prudent.

\section{Data Availability}

All data generated or analyzed during this study are included in this published article.

\section{Consent}

Informed written consent for publication and accompanying images was obtained from the patient prior to collecting information.

\section{Conflicts of Interest}

The authors declare that they have no competing interests.

\section{Authors' Contributions}

$\mathrm{OB}$ and $\mathrm{UJ}$ contributed to the collection of information and writing of the manuscript. NF and SS contributed to the writing and final approval of the manuscript. All authors read and approved the final version of the manuscript.

\section{References}

[1] S. Hirota, K. Isozaki, Y. Moriyama et al., "Gain-of-function mutations of c-kit in human gastrointestinal stromal tumors," Science, vol. 279, no. 5350, pp. 577-580, 1998.

[2] G. L. Ma, J. D. Murphy, M. E. Martinez, and J. K. Sicklick, "Epidemiology of gastrointestinal stromal tumors in the era of histology codes: results of a population-based study," Cancer Epidemiology Biomarkers \& Prevention, vol. 24, no. 1, pp. 298-302, 2015.

[3] T. Tomita, J. Palacherla, M. Zuckerman, S. Dougherty, and M. Ghaleb, "Case report: gastrointestinal stromal tumor of jejunum with angiodysplasia," Digestive Diseases and Sciences, vol. 49, no. 4, pp. 667-671, 2004.

[4] S. H. Shim, Y. H. Han, J. E. Kwak et al., "Synchronous development of gastrointestinal stromal tumor and arteriovenous malformation in the jejunum: a case report," The Korean Journal of Pathology, vol. 42, no. 3, pp. 185-188, 2008.

[5] K. Shiozawa, M. Watanabe, Y. Igarashi et al., "Gastrointestinal stromal tumor mimicking arteriovenous malformation of the jejunum," Case Reports in Gastroenterology, vol. 5, no. 3, pp. 558-564, 2011.

[6] S. J. Javeed, R. Ishaq, A. Khalid, and A. K. Tanwani, "Synchronous development of gastrointestinal stromal tumor and arteriovenous malformation in the jejunum," International Journal of Pathology, vol. 13, pp. 176-178, 2018.
[7] T. Tran, J. A. Davila, and H. B. El-Serag, "The epidemiology of malignant gastrointestinal stromal tumors: an analysis of 1 , 458 cases from 1992 to 2000," American Journal of Gastroenterology, vol. 100, no. 1, pp. 162-168, 2005.

[8] M. Miettinen, H. Makhlouf, L. H. Sobin, and J. Lasota, "Gastrointestinal stromal tumors of the jejunum and ileum: a clinicopathologic, immunohistochemical, and molecular genetic study of 906 cases before imatinib with long-term follow-up," The American Journal of Surgical Pathology, vol. 30, no. 4, pp. 477-489, 2006.

[9] M. Scarpa, M. Bertin, C. Ruffolo, L. Polese, D. F. D'Amico, and I. Angriman, "A systematic review on the clinical diagnosis of gastrointestinal stromal tumors," Journal of Surgical Oncology, vol. 98, no. 5, pp. 384-392, 2008.

[10] C.-M. Lee, H.-C. Chen, T.-K. Leung, and Y.-Y. Chen, "Gastrointestinal stromal tumor: computed tomographic features," World journal of gastroenterology: WJG, vol. 10, no. 16, pp. 2417-2418, 2004.

[11] Division of Digestive Surgery, Y. Mazni, and R. D. K. Robby, "CT scan as a diagnostic modality of gastrointestinal stromal tumor: a systematic review," The New Ropanasuri Journal of Surgery, vol. 3, no. 2, pp. 25-27, 2018.

[12] H. S. Vats, T. J. Wengert, and C. F. Torbey, "Gastrointestinal stromal tumor with Dieulafoy lesion: a novel association," Clinical Medicine \& Research, vol. 4, no. 3, pp. 228-229, 2006.

[13] C. S. Jackson and L. B. Gerson, "Management of gastrointestinal angiodysplastic lesions (GIADs): a systematic review and meta-analysis," American Journal of Gastroenterology, vol. 109, no. 4, pp. 474-483, 2014.

[14] S. Y. Lee, B. K. Goh, E. Sadot et al., "Surgical strategy and outcomes in duodenal gastrointestinal stromal tumor," Annals of Surgical Oncology, vol. 24, no. 1, pp. 202-210, 2017. 